Investigaciones y experiencias 



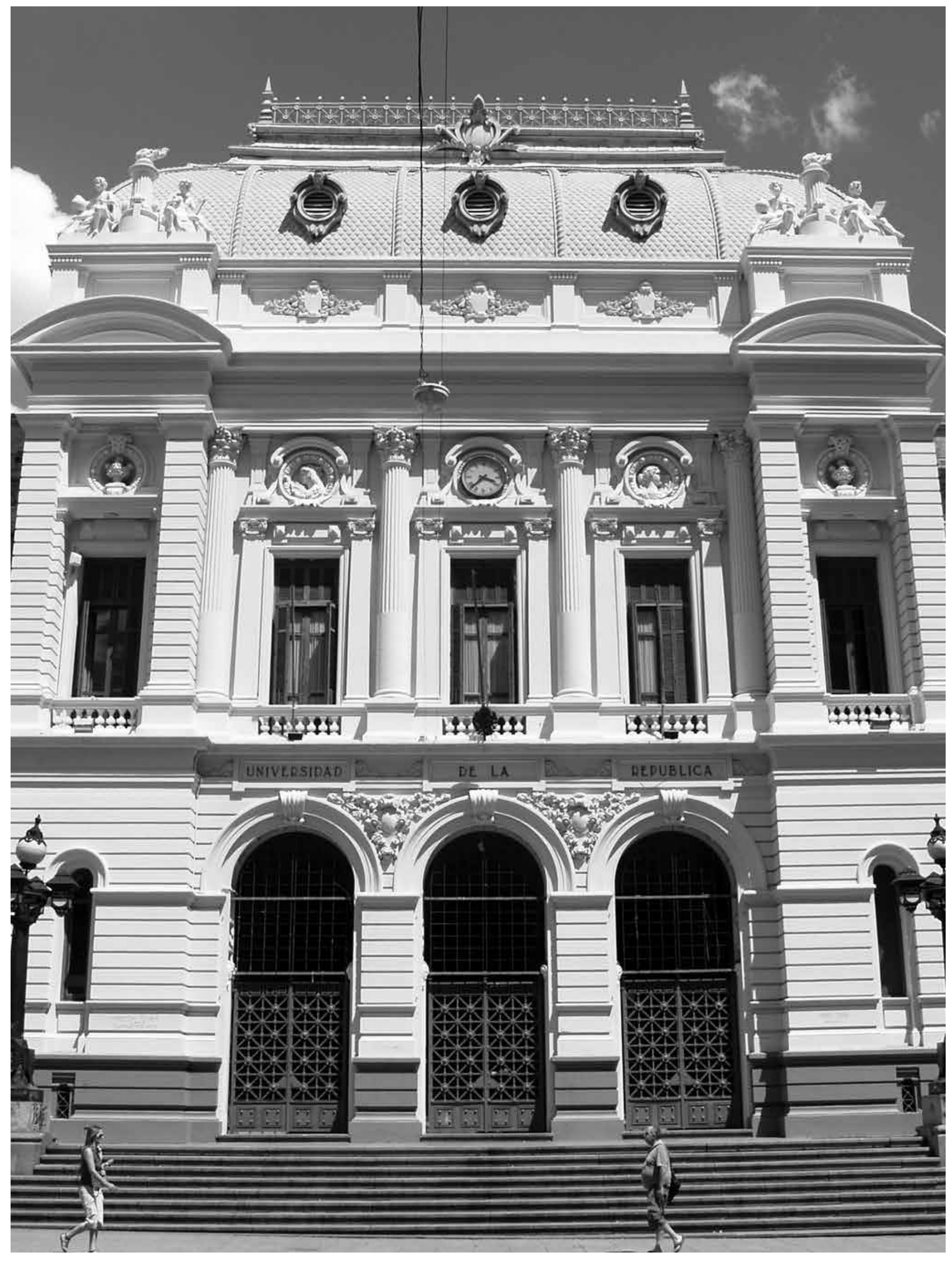




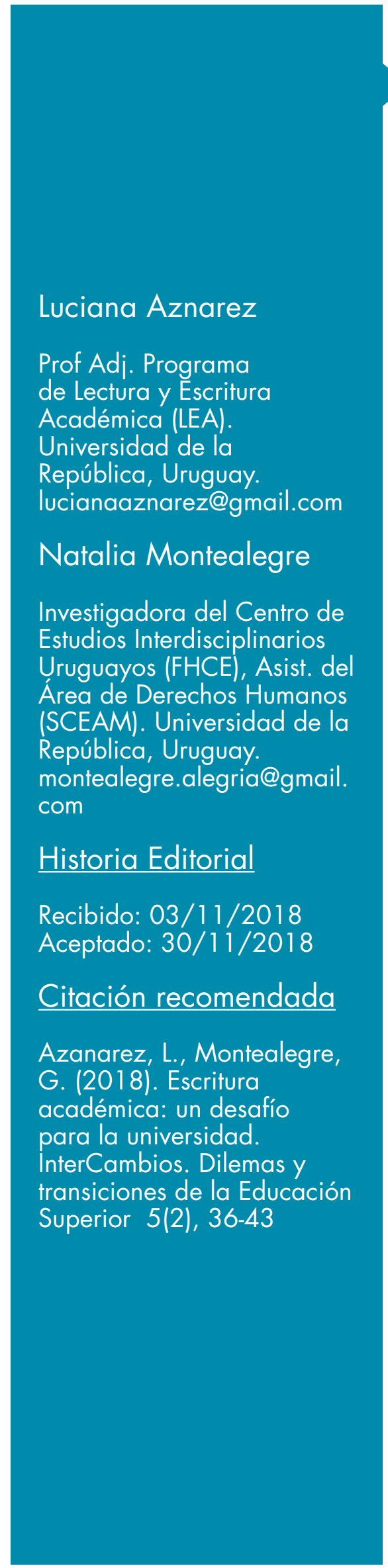

\section{Escritura académica: un desafío para la universidad}

\section{Academic writing: a University challenge Escrita acadêmica: um desafio para a universidade}

Resumen

Este artículo busca compartir algunas reflexiones y aspectos polémicos a la hora de pensar la escritura académica en la Universidad de la República (Udelar). El objetivo es pensar la escritura académica como un escollo para el ejercicio pleno del derecho a la educación en nuestro contexto universitario y desde el punto de vista de todos los actores: docentes, estudiantes y egresados. Para ello, se presentará una definición de alfabetización, alfabetización académica y escritura académica. En segundo lugar, se explicitará una concepción de enseñanza y aprendizaje desde la cual pensar los procesos escriturales en la universidad. En tercer lugar, se aludirá a aspectos relativos al currículo lingüístico. En cuarto lugar, se desarrollarán algunas implicancias evolutivas para el acceso a la escritura. Por último, se reflexionará acerca de la escritura académica vinculada al derecho a la educación universitaria y el rol de la institución para intervenir en la necesaria formación de todos los actores para el desarrollo de la escritura académica.

\section{Palabras claves:}

Universidad, escritura académica, derecho a la educación.

\section{Abstract}

This article discusses some reflections and controversial aspects of academic writing within the Universidad de la República (Udelar). The objective is to think about this issue as a stumbling block for the full exercise of the right to education in our university context and from the point of view of all actors: teachers, students and graduates. It opnes with a brief definition of literacy, academic literacy and academic writing. In the second place, it describes a teaching and learning conception to think the literacy processes in the university. In the third place, reference will be made to aspects related to the linguistic curriculum. Fourth, some evolutionary implications in the writing skills will be developed. Finally, we will reflect on the academic writing linked to the right to university education and the role of the institution to intervene in this process.

\section{Keywords:}

University, academic writing, teachers, students, graduates. 
Resumo

Este artigo procura compartilhar algumas reflexões e aspectos controversos quando se pensa em redação acadêmica na Universidade da República (Udelar). O objetivo é pensar que a escrita acadêmica é um obstáculo para o pleno exercício do direito à educação em nosso contexto universitário e do ponto de vista de todos os atores: professores, alunos e graduados. Para isso, será apresentada uma definição de alfabetização, alfabetização acadêmica e redação acadêmica. Em segundo lugar, uma concepção de ensino e aprendizagem a partir da qual pensar os processos das escrituras na universidade será explicada. Em terceiro lugar, será feita referência a aspectos relacionados ao currículo linguístico. Em quarto lugar, algumas implicações evolutivas para o acesso à escrita serão desenvolvidas. Por fim, refletirá sobre a redação acadêmica vinculada ao direito à formação universitária e o papel da instituição para intervir na necessária formação de todos os atores para o desenvolvimento da escrita acadêmica.

\section{Palavras-chave:}

Universidade, escrita acadêmica, direito à educação.

\section{Presentación}

Existe un sinnúmero de investigaciones que demuestran que los estudiantes que finalizan la secundaria ${ }^{1}$ tienen falencias en las áreas de lectura y escritura y que estas dificultades persisten a lo largo de toda la vida universitaria (Christie, 2012; Gabbiani y Orlando, 2015 y 2016; Giudice y Moyano, 2011; Hernández Ramírez, 2010; Hood, 2010; Mattioli, 2010; Vian Junior, 2011; entre otros). Las distintas teorías sobre alfabetización académica, laboratorios y talleres remediales proponen diversas formas de entender y trabajar con la escritura de textos académicos. Sin embargo, más allá de los aciertos o dificultades de tales o cuales metodologías, existe un punto en el que todas las investigaciones consultadas están de acuerdo, en palabras simples: a escribir se aprende escribiendo a partir de una retroalimentación de calidad, sistemática y sostenida a lo largo de un período prolongado. Esto significa que, además de ser un proceso trabajoso, se necesitan dos actores involucrados: el que escribe y aquel que corrige, guía o enseña.

Este artículo conceptualiza algunos aspectos relativos a la escritura académica, en el entendido de que la falta de un acceso efectivo a esta práctica constituye una de las formas de exclusión educativa con las que convivimos. En ese sentido, y como plantea Rinesi (2015), se problematizará la agenda universitaria referida a la escritura académica, dado que la ausencia del tema en términos de investigación y ajuste curricular se erige como un escollo para el ejercicio pleno del derecho a la educación en el contexto universitario. Se estima que estos aspectos tienen implicancias para el desarrollo académico de los estudiantes, el ejercicio de las profesiones y, especialmente, la consecución de una carrera académica para los docentes.

Con este interés, en primer lugar, se definirá qué se entiende por escritura académica en la universidad. En segundo lugar, se explicitará una concepción de enseñanza y aprendizaje. En tercer lugar, se conceptualizarán aspectos del currículo lingüístico universitario. En cuarto lugar, se desarrollarán algunas implicancias evolutivas para el acceso a la escritura. Por último, se reflexionará acerca de la escritura académica y el derecho a la educación universitaria.

\section{Escritura académica en la univer- sidad}

En términos generales se define la alfabetización o literacidad como un fenómeno social y cultural relacionado con la enseñanza-aprendizajepotenciamiento de diferentes modos del lenguaje: escrito, oral, multimodal, entre otros (Christie, 2012; Schleppegrell, 2004; Rogers, 2004; Kress, 2005). En términos particulares, la alfabetización académica y profesional se relaciona con la enseñanza de contenidos a través de lenguajes disciplinares y géneros discursivos particulares. La escritura académica universitaria es una de las formas en las que los géneros discursivos se cristalizan y puede ser definida como el proceso por el cual diversas comunidades de práctica, en la órbita de la educación terciaria, producen y reproducen textos escritos con diversos propósitos sociales. En este sentido, se entiende que la escritura es una práctica sociosemiótica que emerge en un contexto y que está sociohistóricamente situada (Gee, 2004). Es decir, que configura un campo atravesado por diversas dimensiones, usos y usuarios que la caracterizan, a la vez que la anclan a prácticas concretas, lógicas institu-

1 La ley uruguaya de educación, 18.437, establece la obligatoriedad de la enseñanza hasta el segundo ciclo de educación media en base a los siguientes principios generales: gratuidad, laicidad e igualdad de oportunidades. Asimismo, se crea el Sistema Nacional de Educación en torno al que se espera se nucleen el conjunto de propuestas educativas «integradas y articuladas para todos los habitantes a lo largo de toda la vida» a partir de la concepción de que la educación es un «bien público». 
cionales y momentos específicos. Por este motivo, se considera útil analizar la escritura académica en la universidad incorporando una visión sociocultural (Prior, 2008).

\section{Comunidad de la práctica como} dispositivo de enseñanza y aprendizaje

El aprendizaje del lenguaje escrito en la universidad se puede entender como una participación periférica legítima (PPL) en comunidades de práctica (Wenger, 2006). El concepto de comunidad permite no ceñirse al de disciplina, generando visibilidad sobre la multiplicidad de formas de escritura que existen en estos ámbitos de producción de conocimiento. Asimismo, pone de relieve el carácter cultural y socialmente situado del lenguaje, que junto con el concepto de participación permite dar cuenta del carácter dinámico y cambiante. Esta visión interpela los enfoques centrados en el disciplinamiento, debido a que coloca en el centro del proceso al intercambio aprendiz-experto.

La comunidad de práctica refiere a grupos de personas que comparten una preocupación o una pasión por algo y que aprenden cómo mejorarlo a través de una interacción regular (Wenger, 2006). En el caso de la escritura académica, la comunidad universitaria persigue objetivos diversos que, para su consecución, requieren de diferentes instancias de socialización y explicitación de las normas. En estos procesos de intercambio y de conocimiento de la práctica, los estudiantesnovatos y los docentes-expertos participan en actividades y discusiones, al tiempo que interactúan unos con otros para alcanzar las metas esperadas.

El concepto de participación periférica legítima (PPL) proporciona otro punto de vista analítico para entender el aprendizaje, que se produce desde la periferia hacia el centro. En este sentido, cabe destacar que Gee (2004) señala que la única manera de apropiarse de la escritura «consiste en su- mergirse (a modo de aprendiz) como miembro de una práctica social» (p. 56). Por este motivo, el concepto de PPL se ajusta a la descripción de lo que implica escribir en la universidad, ya que pone de manifiesto el carácter laborioso, sistemático y sostenido a lo largo del tiempo que debe invertir el aprendiz para su aprendizaje. Sin embargo, la participación no es suficiente si no se cuenta con la presencia y el apoyo del otro experto que, mediante la corrección sistemática de diversos textos y en diversas instancias, habilita al estudiante a salir de la periferia para integrar parte del núcleo o centro (Lave y Wenger, 1997).

La perspectiva planteada pone de manifiesto que los significados devienen en un proceso de negociación y renegociación continua, en el que el aprendiz y el experto interactúan en una simultaneidad de roles que conllevan diferentes grados de responsabilidad. La participación activa de ambos posibilita un crecimiento en el conocimiento y, en tanto proceso de cambio, forma parte de la construcción de identidades.

Por todo lo antes dicho, la universidad como institución de educación superior y como productora de conocimiento no puede estar ajena a la calidad de la producción de textos de los participantes involucrados en esos procesos y es, a la vez, responsable por ello. En primer lugar, porque la escritura es la forma más usual para la evaluación del conocimiento de sus estudiantes. En segundo lugar, porque las exigencias para los productores de conocimiento (estudiantes, docentes y egresados) de publicar sus trabajos están ligadas a lógicas globales a las que no todos los universitarios tienen acceso. En tercer lugar, porque la centralidad que cobra el acceso a las prácticas de escritura como elemento distintivo de la educación terciaria entronca su aprendizaje con el ejercicio genuino del derecho a la educación que la universidad debe garantizar.

El ejercicio del derecho a la escritura en la educación superior debería efectivizarse en una oferta de contextos de uso en la que el estudiante pueda participar y tener posibilidades de ser exitoso en sus aprendizajes. De esta manera, elementos valorados en la escritura como la nominalización, la adjetivación, las frases preposicionales y oraciones subordinadas deben ser comprendidos a la luz del contexto de producción (la comunidad de práctica). Asimismo, será necesario dotar a los docentes-expertos de herramientas que les permitan guiar y asesorar a los estudiantes en los procesos de producción de conocimiento ligados a la escritura. Estos son aspectos que forman parte del currículo lingüístico de la Universidad de la República que es preciso revisar y explicitar para que puedan ser incorporados de manera exitosa a las prácticas docentes.

\section{Currículo lingüístico}

La universidad presenta a sus estudiantes una nueva situación comunicativa que es diferente de aquella que transitaron en la enseñanza media. Los ámbitos de enseñanza terciarios no solo introducen nuevas formas de escritura, sino que presentan nuevas formas de interactuar, nuevos tipos de textos, nuevas tareas de lectura y nuevos géneros que construyen nuevas clases de conocimiento. Todos estos aspectos están relacionados con las prácticas de producción de conocimiento necesarias para transitar el proceso hacia la titulación.

La escritura académica forma parte de uno de los requisitos fundamentales para una participación efectiva dentro de la comunidad universitaria. Sin embargo, en muchos casos, las prácticas de escritura no conllevan guías claras sobre cómo se debe estructurar y organizar un texto para ser aceptado como "correcto». Sucede que los patrones esperados de escritura son muchas veces implícitos y de difícil acceso para los no iniciados. Por esta razón, aquellos estudiantes que carecen de un suficiente dominio lingüístico de estas competencias tendrán dificultades en las tareas de escritura y, por ende, en la demostración de sus conocimientos. En este 
Figura 1

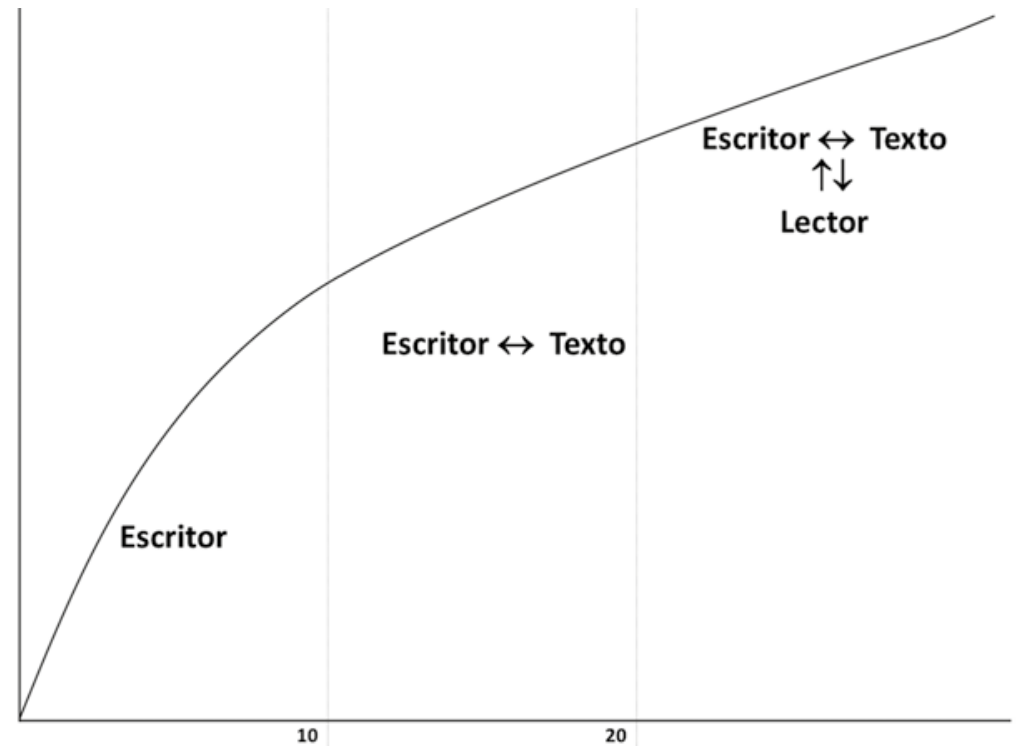

Fuente: Adaptado de Kellogg (2008).

sentido, es posible pensar que las dificultades de razonamiento de muchos estudiantes pueden deberse a una ausencia de familiaridad con las propiedades del lenguaje, a través del cual se espera que realicen el razonamiento, más que a dificultades en el proceso cognitivo involucrado.

El contexto de masificación ${ }^{2}$ de estudiantes que atraviesan muchos servicios universitarios - y algunas carreras en particular- impide la efectivización de prácticas de escritura. Por ejemplo, hay asignaturas cuya evaluación se produce únicamente a partir de parciales o exámenes de múltiple opción. En esos casos, los estudiantes tienen un contacto limitado con el lenguaje académico vinculado solo a la lectura de textos. En este sentido, resulta oportuno advertir el riesgo para la universidad de formar sujetos que no tengan la posibilidad efectiva de participar en suficientes prácticas de escritura guiadas, ya que será el augurio de un fracaso posterior. Este es el caso de muchos alumnos avanzados que ven impedida la finalización de sus estudios debido, por ejemplo, a la incapacidad en la escritura de las monografias finales de grado.

Los estudiantes que no han tenido la oportunidad o han tenido escasas oportunidades de practicar la escritura académica no deberían ser culpabilizados de estos problemas. Si se estima que la escritura es el medio por el cual se transmite el conocimiento en la educación superior y que para un desarrollo y dominio experto se requieren espacios de formación vinculados a las prácticas concretas, es necesario que esta dificultad sirva como indicador de un ajuste curricular necesario.

La explicitación de algunas de las características del currículo lingüístico de la universidad permite generar conciencia sobre el lugar que tiene el lenguaje escrito a la hora de pensar la formación universitaria. Estos aspectos también permiten visualizar el papel y el rol que tiene que jugar la universidad para abordarlos. Sin embargo, un aspecto fundamental para proyectar un plan de trabajo debe provenir del conocimiento de una investigación lingüística sistemática de los géneros escritos (Schleppegrell, 2004) que se exigen en las diversas comunidades de práctica. En palabras de Christie (2012): se necesita una identificación de las características fundamentales de los discursos académicos y de cómo construyen sus estructuras de conocimiento, para que esta información permita desarrollar pedagogías de intervención focalizadas y contextualizadas (2012: 190). Otro aspecto relevante a tener en cuenta para el diseño e implementación de políticas tendientes a fortalecer a nuestra comunidad universitaria en la escritura son los resultados de las investigaciones sobre el desarrollo de la escritura a lo largo de la vida. Como se verá a continuación, proporcionan información acerca de elementos ma-

2 «Masificación» entendida como el incremento en términos absolutos de la matrícula estudiantil, que no tiene correlato en el presente con el magro presupuesto asignado a la Udelar en Uruguay. Por este motivo, el fenómeno de las asignaturas masivas tiende a agravarse en las diversas áreas de conocimiento.Vale tener en cuenta que la cantidad de ingresos de estudiantes en todo el Uruguay ha tenido un crecimiento sostenido significativo. En 2017 ingresaron 17.627 estudiantes, prácticamente un $10 \%$ más que en 2015 y casi 32 \% más que en 2010. Las proyecciones de la División Estadística de la Dirección General de Planeamiento de la Universidad de la República estiman que la cantidad de matriculaciones podría evolucionar hacia 150.000 estudiantes en el año 2019. 
durativos que nos llaman a pensar en lo fundamental de enseñar escritura académica en la universidad.

\section{Implicancias evolutivas en la capacidad de escritura}

La escritura de un texto implica ordenar, jerarquizar y seleccionar las ideas que se desea transmitir a partir del lenguaje como medio y como fin. El proceso de escritura permite a sus usuarios transmitir de maneras diversas una misma idea, lo que demuestra que no se trata solamente del dominio de los conocimientos, sino de una habilidad lingüística para textualizarlos. El desarrollo de la capacidad de expresión escrita evoluciona a través del tiempo y con la práctica y requiere de años de enseñanza y aprendizaje mediante ensayo-error y retroalimentación de calidad.

Kellogg (2008) plantea que el desarrollo de la habilidad de composición de textos evoluciona en tres fases, que se muestran en la figura 1 y que son el resultado de al menos dos décadas de maduración, instrucción y práctica.

La primera fase que se describe y que requiere al menos diez años de desarrollo se denomina decir el conocimiento o knowledge telling. En esta etapa, la representación de la escritura está centrada en el autor y su uso se concibe como medio para expresar lo que uno sabe. La segunda fase o etapa intermedia requiere 20 años de práctica para que el escritor sea capaz de transformar en texto lo que sabe y que esto sea en su beneficio y se denomina knowledge transforming o transformación en conocimiento. La fase final, en la que hay una elaboración de lo que uno sabe para el beneficio del lector, se produce recién cuando han pasado
20 años de práctica. Kellogg afirma que el knowledge crafting o elaboración del conocimiento es una "habilidad avanzada de escritura que requiere una capacitación sistemática, así como instrucción para que la atención ejecutiva pueda coordinar con éxito múltiples procesos de escritura y representaciones simultáneas [autor-texto-lector]» (2008: 22).

Las dos primeras han sido descritas como las que típicamente se adquieren durante la primaria y la secundaria. La última se discute con menos frecuencia porque caracteriza la progresión del profesional hacia una experticia en escritura. Sin embargo, sus implicaciones son esenciales para la educación universitaria porque demuestran que es la instrucción que se produce luego de los 20 años la que permite generar procesos de escritura profesional. Por esta razón, se entiende que la universidad debería ofrecer a sus estudiantes la posibilidad de aprender e insertarse en diferentes prácticas de escritura académicas a lo largo de todo el ciclo universitario.

\section{Escritura académica y derecho a la educación universitaria}

La descripción ad hoc realizada sobre el currículo lingüístico de la universidad, así como la constatación de la ausencia de espacios curriculares generalizados para participar en prácticas de escritura académica dejan planteados algunos interrogantes. Cabe destacar que los espacios son limitados no solo para los estudiantes, sino también para los docentes, a quienes se les exige ser expertos y fomentar la participación de los novatos con relación a los contenidos y a la forma de esos contenidos (o escritura académica). Otro aspecto llamativo es la ausencia de una política central relativa al desarrollo de investigación para relevar las exigencias lingüísticas de los diversos currículos y la falta de medidas centrales extensivas a la totalidad de las áreas de conocimiento que sirvan de apoyo para todos los actores que buscan publicar artículos de calidad, es decir, todos aquellos que quieren el desarrollo de una carrera docente, de investigación y/o de extensión. La explicitación de algunas de las problemáticas pone el foco en una de las causas de la marginación de estudiantes que no acceden a un nivel de escritura acorde, o de los docentes que no pueden publicar, o aun de los egresados, quienes muchas veces no logran niveles de experticia en la escritura en sus prácticas profesionales. Se estima que para mejorar la participación de los actores en prácticas letradas dentro de la comunidad universitaria hay que generar espacios, así como hacer alianzas con aquellos docentes que se sientan capacitados para formar, además de en contenidos, en escritura. Para ello, existen algunas experiencias de enseñanza específicas que deberían ser evaluadas para identificar sus potenciales aportes en los ámbitos institucionales más diversos. Asimismo, se resalta la necesidad de investigación sistemática, que permita entender cómo se construye la desventaja que presentan algunos estudiantes y docentes en su adaptación al lenguaje académico y que, a su vez, permita buscar alternativas para eliminarla. Se acuerda con Rinesi (2015) que será necesario un reposicionamiento de la actividad docente, ya que es en el fortalecimiento de las prácticas formativas que se contribuye a garantizar el derecho a la educación universitaria en toda su extensión. 


\section{Referencias bibliográficas}

Christie, F. (2012). The Overall Trajectory in Language Learning. Chapter 6. En Language Education Throughout the School Years: A Functional Perspective. Oxford, Malden:Wiley-Blackwell, 187-224.

Gabbiani, B. y Orlando, V. (orgs.) (2015). Escritura, lectura y argumentación en contextos educativos del Río de la Plata. Montevideo: Ediciones Universitarias (Ucur).

- (comp.) (2016). Escritura, lectura y argumentación en las monografías de humanidades. Montevideo: Udelar, CSE.

GeE, J. P. (2004). Oralidad y literacidad: de El pensamiento Salvaje a Ways with Words. En V. Zavala, M. Niño-MurCia y P. Ames (2004) (eds.). Escritura y sociedad. Nuevas perspectivas teóricas y etnográficas. Lima: Red para el Desarrollo de las Ciencias Sociales en el Perú, 23-55.

Giudice, J. y Moyano, E. (2011). Apropiación del discurso de la economía: análisis evolutivo de un caso. En Barbara, Leila y E. Moyano. Textos y lenguaje académico. Exploraciones sistémico-funcionales en portugués y español. Buenos Aires: Universidad Nacional de General Sarmiento.

Hernández Ramírez, L. (2010). Estructura esquemática y condiciones de cohesión en el discurso especializado médico: el caso de la nota médica. En D. FernándeZ y E. GHio (comp.), El discurso en español y portugués. Estudios desde una perspectiva sistémico-funcional. Universidad Nacional del Litoral.

Hood, S. (2010). Appraising research. Evaluation in Academic Writing. Londres: Palgrave Macmillan.

KellogG, R. T. (2008). Training writing skills: a cognitive developmental perspective. Journal of writing research, 1 (1), $1-26$.

Kress, G. (2005). El alfabetismo en la era de los nuevos medios de comunicación. Andalucía: Enseñanza abierta de Andalucía.

Lave,J. y Wenger, E. (1997). Situated learning. Legitimate periphereal participation. Nueva York: Cambridge University Press.

Mattioli, E. I. (2010). Las estrategias de expansión y abstracción en los textos que reciben y producen los alumnos ingresantes a la universidad. En D. FERNÁNDEZ y E. GHio (comp.), El discurso en español y portugués. Estudios desde una perspectiva sistémico-funcional. Universidad Nacional del Litoral.

Prior, P. (2008). A Sociocultural Theory ofWriting. En C.A. MacArthur, S. Graham \& J. Fitzgerald (2008), Handbook of writing research. Nueva York: Guilford Press, 54-66.

Rinesi, E. (2015). Filosofía (y) politica de la universidad. Buenos Aires. Los Polvorines, Universidad Nacional de General Sarmiento.

Rogers, R. (2004). An Introduction to Critical Discourse Analysis in Education. En R. Rogers (2004), An Introduction to Critical Discourse Analysis in Education. New Jersey. Lawrence Erlbaum Associates, Inc.

Schleppegrell, M. (2004). The Language of Schooling. A Functional Linguistics Perspective. Londres: Routledge.

VIAN, O. (2011). O artigo na familia de generos académicos: notas sobre aspectos tipológicos, topológicos e seu papel no ensino-aprendizagem de leitura. En Barbara, Leila y E. Moyano (2011), Textos y lenguaje académico. Exploraciones sistémico-funcionales en portugués y español. Buenos Aires: Universidad Nacional de General Sarmiento.

Wenger, E. (2006). Communities of practice. A brief introduction. Disponibles en http://www.ewenger.com/theory/> (6 de julio de 2012). 


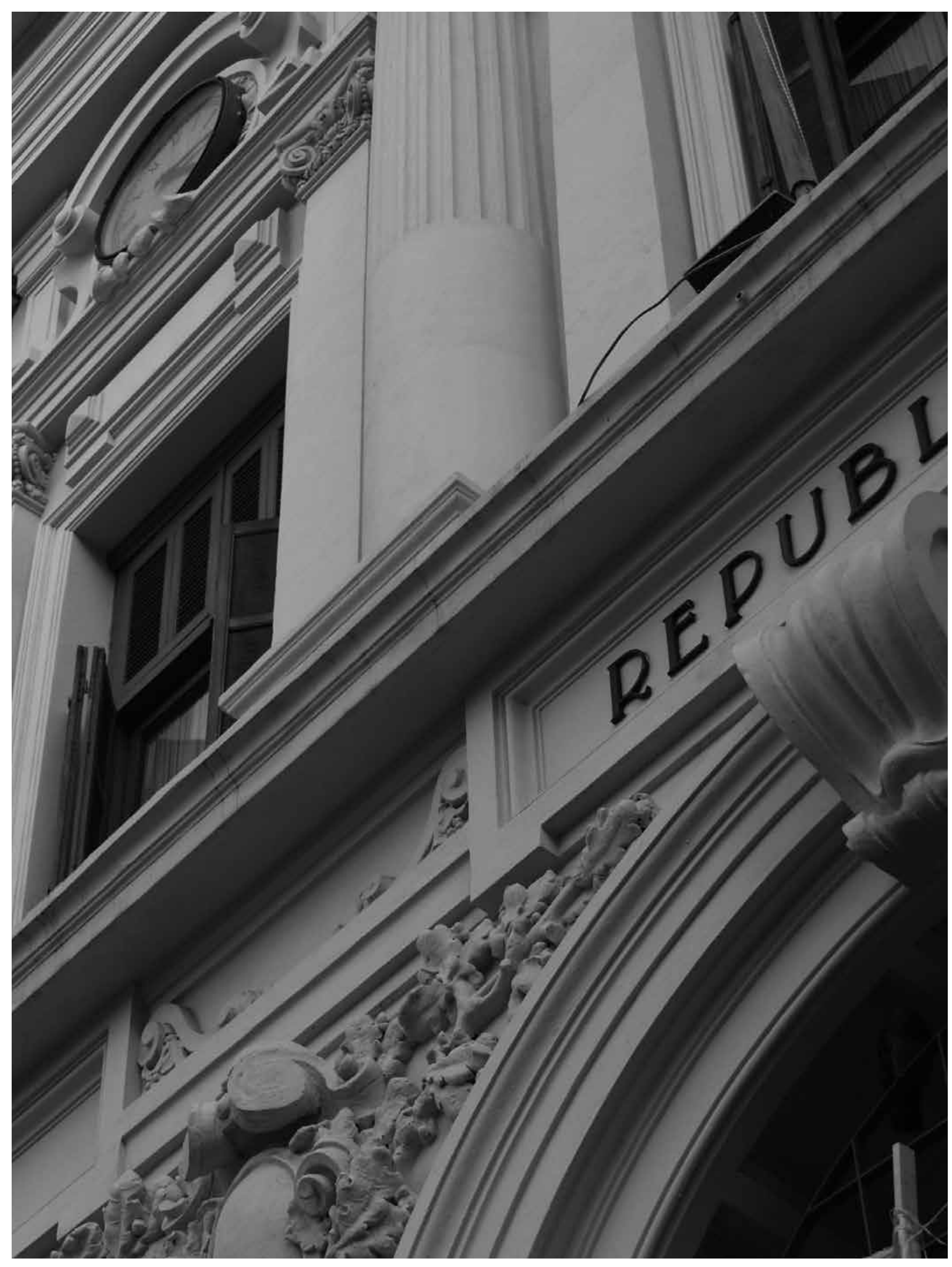

